\title{
Dynamics of the CapG actin-binding protein in the cell nucleus studied by FRAP and FCS
}

\author{
Malte Renz ${ }^{1,2} \&$ Jörg Langowski ${ }^{1 *}$ \\ ${ }^{1}$ German Cancer Research Center, Division Biophysics of Macromolecules, Im Neuenheimer Feld 580, \\ D-69120 Heidelberg, Germany; E-mail: jl@dkfz-heidelberg.de; ${ }^{2}$ Department of Obstetrics and Gynecology, \\ Heinrich-Heine-University Düsseldorf, D-40225, Düsseldorf, Germany \\ * Correspondence
}

Key words: actin-binding protein, breast cancer, intranuclear mobility, live cell analysis

\begin{abstract}
FRAP (fluorescence recovery after photobleaching) and FCS (fluorescence correlation spectroscopy) are spectroscopic methods for monitoring the dynamic distribution of proteins inside the nucleus of living cells. As an example we report our studies on the intracellular mobility of the actin-binding protein CapG in live breast cancer cells. This Gelsolin-related protein is a putative oncogene. It appears to be overexpressed especially in metastasizing breast cancer. Furthermore, the CapG protein is known to be involved in the motility control of non-muscle benign cells. Its increased expression triggers an increase in cell motility of benign cells. Thus it can be expected that in cancer cells overexpressing the CapG protein, motility, invasiveness and metastasis might be particularly promoted. Since the nuclear CapG fraction seems to be pivotal to the increase in cell motility, we focused our studies on the CapG mobility in cell nuclei of live breast cancer cells. Using FCS and FRAP we showed that the eGFP-tagged CapG is monomeric and characterized its diffusional properties on the microsecond to minute timescale. This information about the mobility and compartmentalization of CapG might help to provide insight into its function within the cell nucleus and give clues about its altered cellular function in malignant dedifferentiation.
\end{abstract}

\section{Abbreviations}

$\mathrm{ACF}$

CapG

CapG-eGFP

eGFP

FCS

FRAP

NLS

PI 3-kinase

$\mathrm{PIP}_{2}$-binding autocorrelation function Gelsolin-related actin binding capping protein CapG-eGFP fusion protein enhanced green fluorescent protein fluorescence correlation spectroscopy fluorescence recovery after photobleaching nuclear localization signal phosphatidylinositol 3-hydroxy kinase phospatidylinositol 4,5-bisphosphate

\section{Introduction}

The interphase cell nucleus forms a highly entangled network of chromatin chains; taking the $30 \mathrm{~nm}$ fibre as the fundamental unit, the volume occupation is about 5-10\% (Weidemann et al. 2003). Proteins and other macromolecules have to be transported through this network in order to access their targets or to leave their site of action. As estimated from the volume occupation, the mesh size of the chromatin 
tangle is of the order of $30-50 \mathrm{~nm}$, leaving ample space for the diffusion of medium-size proteins. In general, proteins have been shown to move through the cell nucleus by diffusional motion that is more or less anomalous: the mean-square displacement of the particle $<r^{2}>$ does not increase linearly with time but with a power law $<r^{2}>\propto t^{\alpha}$, with $\alpha<1$ (Wachsmuth et al. 2003). This 'diffusion anomaly' is influenced by the distribution and density of obstacles in the nucleus - the main one being the chromatin network - and by transient binding to these structures. Units larger than the mesh size, e.g. PML bodies, can also move through the nucleus by a combination of local random motion and longer-distance 'jumps' (Goerisch et al. 2005); in this case, the dynamics of the chromatin network itself have a fundamental role in the mobility of such complexes.

The analysis of the random motion of proteins in the cell nucleus is thus an important tool for understanding their mechanism of action, such as finding a specific target, shuttling between different nuclear compartments, transport into and out of the nucleus and availability of a bound fraction. A number of experimental techniques exist that can visualize the motion of proteins in a cell, most of them based on fluorescent labelling of the moving object. The study that we show here uses fluorescence recovery after photobleaching (FRAP) and fluorescence correlation spectroscopy (FCS) to analyse the mobility of CapG, an actin-binding protein that seems to play a pivotal role in cell mobility and tumour cell invasiveness.

Both methods of kinetic analysis applied here, FRAP and FCS, are experimentally and theoretically related: The numbers of molecules specified by autofluorescence or fluorescent labelling are measured in a defined open observation volume as functions of time, taking advantage of the specificity and sensitivity of fluorescence spectroscopy. Both FRAP and FCS can be used to determine rates of transport processes or chemical reactions, respectively (Elson 1986; Petersen \& Elson 1986).

FRAP is based on the fact that a fluorescent dye can be destroyed or deactivated by high intensity of the excitation light; the zone in which this 'bleaching' occurs is then monitored at a much lower light intensity (typically $0.1-1 \%$ of the bleaching intensity) and the recovery of fluorescence by fluorescent molecules moving into the bleached region and bleached molecules moving out of it is observed and analysed quantitatively. Modern confocal laser scanning microscopes allow easy programming of an arbitrary region in which the scanning laser beam is switched to a high intensity to perform the bleaching, and then the fluorescence recovery in this region is monitored by rapidly acquiring a series of images. The recording frequency of the confocal scans (typically several scans per second, up to about $100 \mathrm{~s}^{-1}$ for the most advanced microscopes) and the diffusion time of the fluorescent molecules into the bleached region determine the timescale of a FRAP experiment, which is of the order of one-tenth of a second to several seconds. Over this timescale, FRAP can be used to determine the characteristic diffusion time of molecules into the bleached region and the fraction of mobile and immobilized species (Axelrod et al. 1976; LippincottSchwartz et al. 2001).

FCS, on the other hand, monitors molecules as they diffuse through a stationary laser focus. The focal volume is so small (of the order of $1 \mathrm{fl}$ ) that only a few molecules are present in the focus at any given time, so that their random motion into and out of the focus causes significant fluctuations in the observed fluorescence intensity. Thus, FCS analyses spontaneous, stochastic intensity fluctuations triggered by single molecules in thermodynamic equilibrium, whereas FRAP monitors the recovery of the initially perturbed steady-state distribution of entire fluorescent and bleached molecule populations. The regression back to equilibrium of spontaneous microscopic fluctuations and the regression of macroscopic displacements from equilibrium are governed by the same phenomenological laws. The stochastic character of the observed intensity fluctuations in FCS, however, requires statistic analysis of a multitude of fluctuations, while in principle a single FRAP observation of entire molecule populations is sufficient to determine transport or reaction constants (Petersen \& Elson 1986). In FCS, the statistical analysis of autocorrelation of the random fluctuations will yield the diffusion coefficient and the concentration of independently diffusing molecules and their association status (Elson \& Qian 1989; Vukojevic et al. 2005). It can also be used to separate diffusion processes that occur on different timescales, such as the motion of small and large fluorescent molecules in a mixture, or free and bound states of the same molecule. Furthermore, diffusion inside random structures (such as the chromatin 
network) will give rise to an apparently timedependent diffusion coefficient, because the probability that a molecule 'gets stuck' during its motion increases with time (Wachsmuth et al. 2000). Thus, FCS offers the opportunity to characterize the Brownian motion of diffusing molecules in great detail. However, FCS is limited to objects that actually move with time, because stationary structures (which are readily characterized by FRAP as immobilized fraction) will not cause fluorescence fluctuations. Thus, the timescales on which information is delivered by FCS range from tens of microseconds to some 100 milliseconds and are complementary to the longer timescales, i.e. some 100 milliseconds to immobilized, monitored by FRAP.

Here we show measurements of the in vivo intranuclear mobility of the actin-binding protein CapG, which seems to play a pivotal role in cell mobility and tumour cell invasiveness.

Initially, a short summary of known CapG properties is given. In what follows, the FRAP and FCS measurements of a CapG-eGFP construct in breast cancer cells are described. These methods provide complementary information about intranuclear CapG distribution, forming the basis for comparative kinetic analyses of normal and neoplastic cells, an example of which we recently published (Renz et al. 2007). In that work we concentrated on FRAP studies of the transport of CapG into the nucleus and could show that in neoplastic cells the recovery rate of CapG was greatly enhanced and the immobilized fraction reduced relative to normal cells. Here we use FCS to further characterize the mobility of CapG inside neoplastic cell nuclei, using the same cell line as in our previous paper.

\section{Known CapG properties}

The CapG protein is a Gelsolin-related actin-binding protein, which is listed as a putative oncogene. It is overexpressed in breast cancer (Dahl et al. 2005), especially in metastasizing breast cancer (D. Niederacher, unpublished data). Furthermore, the CapG protein is known to be involved in the motility control of non-muscle benign cells. Its increased expression triggers an increase in cell motility of benign cells. Thus it can be expected that in cancer cells, which overexpress the CapG protein, motility, invasiveness and metastasis might be particularly promoted.

The relatively small, monomeric CapG (39 kDa) is distributed diffusively throughout the cell, in the cytoplasm and in the nucleus (Onoda et al. 1993). There is no typical nuclear localization signal (NLS), rather several regions of different subdomains are responsible for nuclear import (Gettemans et al. 2005). In contrast to the other Gelsolin-related actin-binding proteins the CapG protein lacks a nuclear export sequence (van Impe et al. 2003). Thus it is the only member of this family that accumulates in the nucleus.

A function that CapG fulfils in the cytoplasm is well known: It blocks the rapidly growing ends of actin filaments (capping) regulated by intracellular $\mathrm{Ca}^{2+}$ and $\mathrm{PIP}_{2}$ concentrations. Thus it is involved in the control of actin-based cell motility (Silacci et al. 2004) and membrane ruffling (phagocytosis) (Witke et al. 2001) of non-muscle cells. It was originally isolated from the cytoplasm of alveolar macrophages (Southwick \& DiNubile 1986), the prototype of a motile and phagocytotic cell. Knockout of CapG causes membrane ruffling and phagocytosis defects in macrophages and motility defects in neutrophil granulocytes and dendritic cells (Parikh et al. 2003), whereas its overexpression triggers an increase in cell motility of various benign cells (fibroblasts (Sun et al. 1995), endothelial cells (Pellieux et al. 2003) and kidney cells of the dog (De Corte et al. 2004)). Some authors refer to the 'established role of CapG in physiological motility of benign cells' (Thompson et al. 2007).

The function of nuclear CapG is largely unknown. Recent studies, however, hint at the fact that the CapG fraction that is localized within the cell nucleus is crucial to cell motility, or rather for the increase in motility of benign cells:

- The induced overexpression of the CapG-eGFP fusion protein in the cytoplasm and in the nucleus triggers an increase in cell motility, whereas overexpression of the protein only in the cytoplasm-by tagging a nuclear export sequence-does not alter the motility of these cells (De Corte et al. 2004).

- Endothelial cells exposed to unidirectional shear stress exhibit an increased ability to migrate and express more CapG: The actin-associated CapG in the cytoplasm doubles within $2 \mathrm{~h}$; the CapG 
localized in the cell nucleus quadruples within 24 h (Pellieux et al. 2003).

Even in cancer cells, nuclear CapG could be decisive for promoting motility, invasiveness and metastasis: In pancreatic cancer, which is reported to overexpress CapG, the intensity of immunohistochemical CapG staining within the cell nucleus appears to be correlated with tumour size. Further correlations between immunohistochemical staining and established prognostic parameters could not be identified (Thompson et al. 2007).

Despite these hints, the actual function of the CapG protein in the cell nucleus remains elusive. Some direct or indirect DNA interaction of CapG was hypothesized: The sequence of the CapG-cDNA shows some overlap with the sequence of a group of DNA-binding proteins, the basic helix-loop-helix family, including the c-myc oncogene. Therefore, the CapG protein was called mbh-1 (myc basic motif homologue-1) (Prendergast \& Ziff 1991). Actual CapG-binding to DNA has not been verified so far. A transactivation activity of the CapG protein was called into question, and a modulation of transcriptional activators was discussed (De Corte et al. 2004).

Phosphorylated CapG localized in the cell nucleus is reported to impair the function of the nuclear PI 3-kinase of fibroblasts of rats and mice in terms of $\mathrm{PIP}_{2}$-binding (substrate-sequestration) (Lu et al. 1998). The PI 3-kinase phosphorylates $\mathrm{PIP}_{2}$, PIP and PtdIns to create potential second messengers regulating DNA synthesis, directly or indirectly.

Several studies have provided evidence that (likely monomeric) nuclear actin associates with three types of nuclear complexes involved in gene expression: chromatin remodelling complexes, ribonucleoprotein particles, and the three RNA polymerases. CapG as a nuclear actin-binding protein could play a role in preventing nuclear actin from polymerizing. Thus it could have some indirect influence on the regulation of gene expression.

Furthermore, an interaction with steroid receptors is possible, which is reported with respect to other Gelsolin-related proteins (Gettemans et al. 2005).

Using spectroscopic methods, i.e. FCS and FRAP, we attempted to characterize the intracellular mobility of this protein in live breast cancer cells. As the nuclear fraction seems to be pivotal to the increase in cell motility, we focused our studies on the CapG mobility in cell nucleus.

\section{Characterization of CapG mobility in breast cancer cell nuclei}

For kinetic analysis in living breast cancer cells, the human CapG protein was C-terminally labelled with eGFP which alters neither the localization of the protein nor its function in the context of cell motility, as reported in our earlier work (Renz et al. 2007). Live cell measurements, FRAP and FCS, were performed at $37^{\circ} \mathrm{C}$ in Hepes-buffered medium or $5 \% \mathrm{CO}_{2}$ atmosphere, respectively.

\section{FRAP — "slow" kinetics of entire CapG populations}

To perform FRAP experiments, a region exposed to high laser intensity was chosen so that the entire cell nucleus was bleached homogeneously. After bleaching, the increase in fluorescence intensity

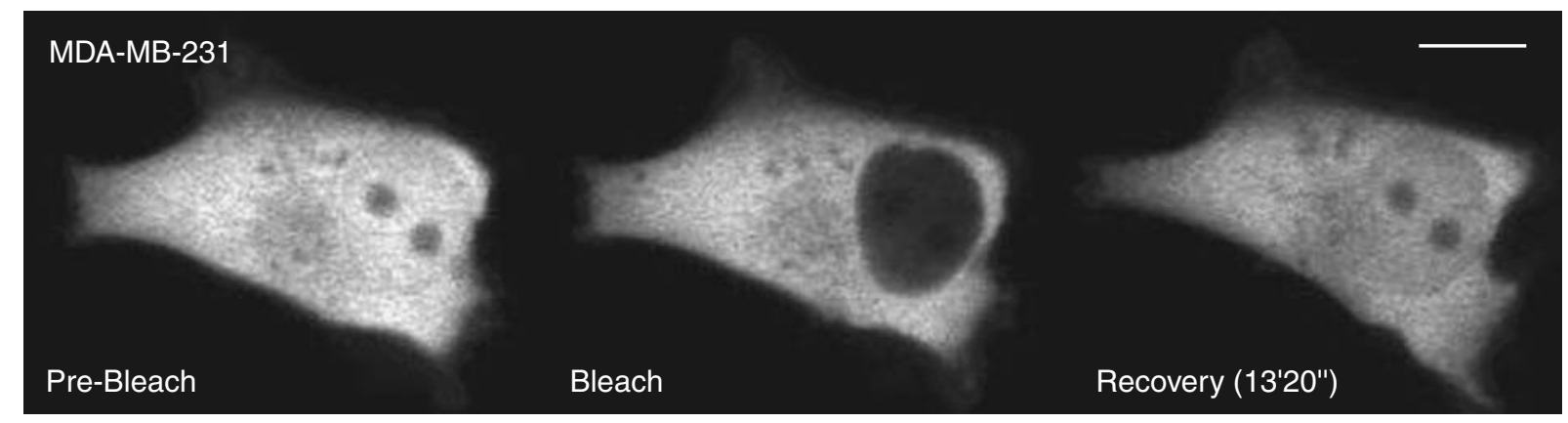

Figure 1. Highly invasive, metastasizing breast cancer cells, MDA-MB-231, transiently transfected with CapG-eGFP. Images were taken before and immediately after bleaching and at the end of the observation period $(13 \mathrm{~min} 20 \mathrm{~s})$. Scale bar $=10 \mu \mathrm{m}$. 
corresponding to the net-uptake of fluorescent CapGeGFP fusion protein into the nucleus was monitored over time by taking a picture every $10 \mathrm{~s}$, in total 80 pictures in 13 min (Figure 1). Potential photobleaching reversibility was improbable owing to the long observation period-reversible dark states due to triplet sequestration occur on a timescale of microseconds to milliseconds. Furthermore, we used a constant acquisition frequency in order not to alter the fraction of potential light-dependent reversible dark states of the eGFP protein.

For quantitative analysis, regions were defined on the acquired images delineating the bleached nucleus, the cytoplasm and the extracellular space. By integrating over all pixels using the image processing software ImageJ (NIH, Bethesda, MD, USA), the fluorescence intensity was determined for each time point in each region. The determined mean grey values were background subtracted, corrected for acquisition photobleaching, laser intensity fluctuations and loss of fluorescence due to the bleaching event. The data was normalized to the initial fluorescence intensity using the following equation (Rabut \& Ellenberg 2005):

$f(t)=\frac{\operatorname{Roi}(t)-\mathrm{BG}(t)}{\operatorname{Tot}(t)-\mathrm{BG}(t)} \times \frac{\operatorname{Tot}(0)-\mathrm{BG}(0)}{\operatorname{Roi}(0)-\mathrm{BG}(0)}$

where $\operatorname{Roi}(t)$ denotes the fluorescence intensity at time $t$ in the nucleus (region of interest), $\operatorname{Tot}(t)$ denotes the fluorescence intensity in the cytoplasm at each time point and $\mathrm{BG}(t)$ is the extracellular background fluorescence in each time point. This analysis results in the graphs in Figure 2.

The normalized fluorescence intensity $f(t)$ was fitted with a single-exponential function:

$f(t)=1-\left[a-b\left(1-\mathrm{e}^{-\lambda t}\right)\right]$

where $a$ is the fraction of initial fluorescence intensity that was bleached, $b$ is the fraction of bleached fluorescence intensity recovered after time $t$ and $\lambda$ is the recovery rate. For the averaged recovery curve of the breast cancer cells we obtain $a=0.783 \pm 0.001$; $b=0.757 \pm 0.006 ; \lambda=(1.373 \pm 0.020) \times 10^{-3} \mathrm{~s}^{-1}$.

Two characterizing kinetic parameters can be determined. (i) The recovery time $\tau$, the reciprocal of the recovery rate $\lambda$, is directly related to the characteristic time for transporting the fluorescent CapG-eGFP fusion protein from the cytoplasm into the cell nucleus. It is $\tau=730 \pm 11$ s. (ii) The immobilized or extremely slowly exchanging fraction of the CapG-eGFP fusion protein within the nucleus can be derived by the difference of the fraction of initial fluorescence bleached, $a$, and the fraction of bleached fluorescence recovered, $b$, namely, $I_{\mathrm{f}}=a-b$. Its value is $3.3 \pm 1 \%$.

In order to analyse whether this immobile component is statistically independent of the recovery time,

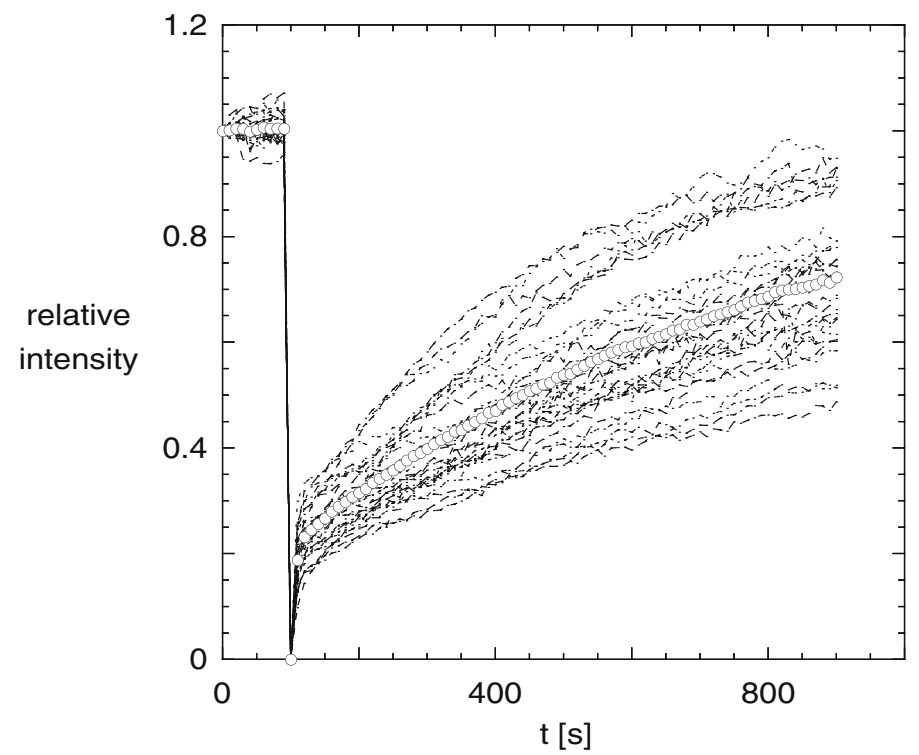

Figure 2. Recovery profiles of 28 MDA-MB-231 cells and corresponding average-value curve (open circles). 

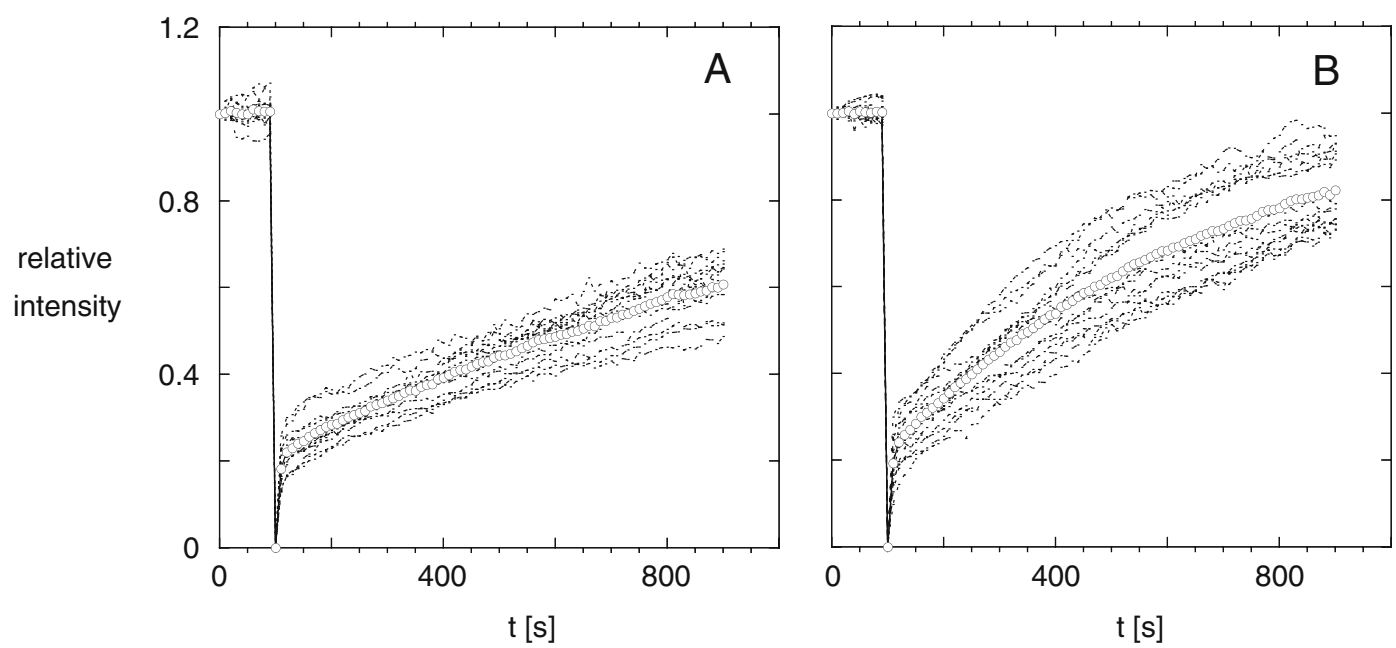

Figure 3. Subgroups of the breast cancer cells (MDA-MB-231) and corresponding average-value curves (open circles): (A) subgroup of relatively slowly recovering cells; (B) subgroup of relatively fast recovering cells.

the cells examined were divided into two groups of equal size according to their recovery rate: one group of relatively fast recovering cells and one of slowly recovering cells (Figure 3). As expected, the recovery times of these two groups diverge, whereas the immobile component appears to be still in the same range (Table 1).

Normal breast epithelium cells exhibit a much higher fraction of immobilized component (Renz et al. 2007). Given the fit uncertainty, the immobile fraction in the neoplastic cells is negligible for both the slow and the fast subgroup. We conclude that the immobilization status, related to the binding properties of the CapG-eGFP fusion protein within the cell nucleus, does not correlate with the recovery time of the observed cells but is a statistically independent parameter characterizing CapG mobility in cancer cell nuclei.

\section{FCS - 'fast' kinetics of few CapG molecules}

\section{Calibration measurements}

To adjust the optical set-up, a calibration series is performed using a $68 \mathrm{nM}$ aqueous solution of Alexa 488. The experimentally obtained autocorrelation functions $G(\tau)$ were fitted with a theoretical model function describing free 3-dimensional diffusion. Two contributing components were taken into account, one non-diffusing, i.e. the triplet state, and one diffusing component:

$$
\begin{aligned}
G(\tau)= & 1+\frac{1}{N}\left(1+\beta \mathrm{e}^{-\lambda \tau}\right)\left(1+\frac{\tau}{\tau_{\text {diff }}}\right)^{-1} \\
& \times\left(1+\frac{\tau}{\tau_{\text {diff }} \kappa^{2}}\right)^{-1 / 2}
\end{aligned}
$$

Here $N$ is the average number of molecules in the focus, $\beta$ and $\lambda$ are the amplitude and decay time of the triplet component, $\tau_{\text {diff }}$ is the diffusion time and $\kappa$ is the axial ratio of the laser focus.

An example: Fitting the experimental autocorrelation function yields the diffusion time $(\tau \approx 37 \mu \mathrm{s})$, the average number of independent diffusing particles in the detection volume $(N \approx 12.5)$ and the $\kappa$-factor $(\kappa \approx 5.5)$, determining the ratio of the axial to lateral radius of the detection volume.

Then, the lateral radius $\left(w_{0}\right)$ of the detection volume can be approximated via the measured diffusion time, using $D_{\mathrm{a} 1488}$ of $300 \mathrm{\mu m}^{2} / \mathrm{s}\left(w_{0} \approx\right.$

Table 1. Comparison of recovery time and immobile component in the examined breast cancer cells

\begin{tabular}{lcc}
\hline Group & $\tau(\mathrm{s})$ & $I_{\mathrm{f}}(\%)$ \\
\hline Slow subgroup & $1134 \pm 71$ & $1.4 \pm 4$ \\
Fast subgroup & $388 \pm 15$ & $-1.6 \pm 2.4$ \\
Entire group & $730 \pm 11$ & $3.3 \pm 1$ \\
\hline
\end{tabular}


$211 \mathrm{~nm}$ ) and via the average particle number, using $\mathrm{\kappa}$ of $5.5\left(w_{0} \approx 214 \mathrm{~nm}\right)$. (The diffusion coefficient of Alexa 488 mentioned in the literature ranges from 200 to $400 \mu \mathrm{m}^{2} / \mathrm{s}$. Measurements at room temperature in comparison to Rhodamine $6 \mathrm{G}$ favour a $D_{\mathrm{a} 1488}$ of $300-320 \mu \mathrm{m}^{2} / \mathrm{s}$.) Thus the diameters of the effective detection volume in this experimental set-up are about $430 \mathrm{~nm}$ in width and $2.4 \mu \mathrm{m}$ in height. Considering the dimensions of a cell nucleus of about $10 \mu \mathrm{m}$ diameter provides an illustrative impression of the detection volume relative to the cell nucleus.

\section{Mobility of inert eGFP in living cells}

Six measurements of $10 \mathrm{~s}$ each were collected with the laser power set to $3.2 \mu \mathrm{W}$, determined at the objective lens. Applied to the effective geometry of the focused laser beam this corresponds to an average power density in the sample of about $3.6 \mathrm{~kW} / \mathrm{cm}^{2}$. Under these conditions, a good signal-to-noise ratio was achieved (molecular brightness of eGFP molecules of about $2 \times 10^{3} \mathrm{cps} /$ molecule) without any photobleaching.
The experimentally derived autocorrelation functions were fitted with a theoretical model function of one diffusing and one non-diffusing component (triplet term) fixed to $30 \mu \mathrm{s}$. Using two diffusing components does not improve the $\chi^{2}$-values and residuals of the fit (Figure 4). The inert eGFP appears to diffuse freely through the cell. Its diffusion time is determined only by the size of the molecule, the temperature and viscosity of surrounding cytoplasm and nucleoplasm, respectively. Transient binding to cellular structures, which would slow down eGFP diffusion and result in a second slowly diffusing component of the ACF, seems to be improbable. The diffusion coefficients in the nucleus and cytoplasm were determined as $D_{\text {GFPnucleus }}=28.5 \pm 0.3 \mu \mathrm{m}^{2} / \mathrm{s}$ and $D_{\text {GFPctoplasm }}=25.8 \pm 0.4 \mu \mathrm{m}^{2} / \mathrm{s}$.

\section{Mobility of CapG-eGFP in breast cancer cells}

After calibration of the system, the kinetics of few CapG-eGFP molecules in living breast cancer cells were characterized.

Representative normalized autocorrelation functions of eGFP and CapG-eGFP in cytoplasm and

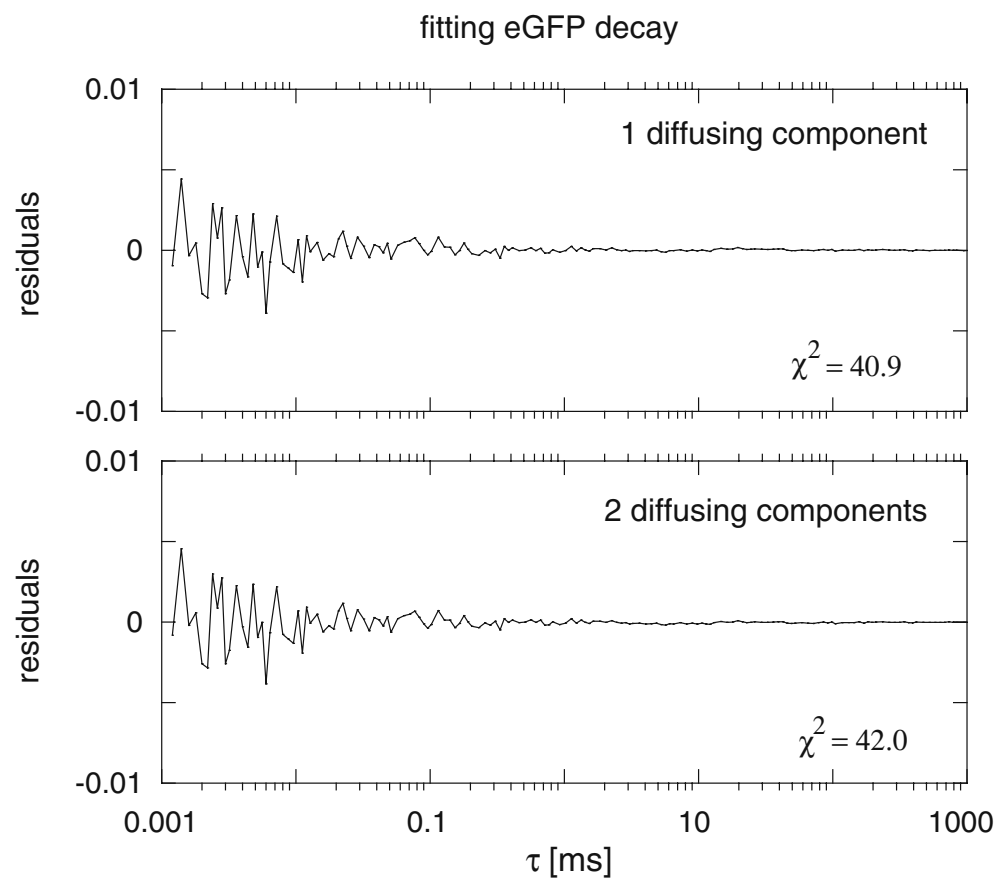

Figure 4. Residuals and $\chi^{2}$-values on fitting with one and two diffusing species, respectively: fitting two diffusing species does not improve fit results of inert eGFP. 
nucleus are shown in Figure 5. Different decay characteristics are apparent. As these measurements are obtained from the same measurements series, the same experimental set-up and optical alignment and the same cell type, the differences shown can be ascribed to the functional component of the CapGeGFP fusion protein.

In contrast to the autocorrelation functions of the inert eGFP, the CapG-eGFP data are described inadequately with a theoretical function considering only one diffusing component. Taking two diffusing components into account, $\chi^{2}$-values and residuals are significantly reduced and the experimental autocorrelation functions are far better approximated (Figure 6). Thus fluorescence correlation spectroscopy detects two diffusing components of CapG-eGFP moving distinctly: the first fast diffusing component characterizes the diffusion of the unbound CapG protein, determined by its molecular dimensions, the temperature and viscosity of the surrounding cellular environment; whereas the second slowly diffusing component describes a CapG fraction which is transiently associated to cellular structures. The fast diffusing component in the nucleus was $D_{\text {CapG-eGFPnucleus }}=22.7 \pm 0.3 \mu \mathrm{m}^{2} / \mathrm{s}$ $(n=41)$ and in the cytoplasm $D_{\text {CapG-eGFPcytoplasm }}=$ $23.9 \pm 0.3 \mu \mathrm{m}^{2} / \mathrm{s}(n=48)$.

Assuming globular proteins, the diffusion coefficient would be inversely proportional to the cube root of their molecular weight. Thus the expected diffusion coefficient of CapG-eGFP can be estimated, relating the molecular weight of free eGFP $(27 \mathrm{kDa})$ to that of the fusion protein $(66 \mathrm{kDa})$; in this case the expected diffusion coefficient would be $D_{\text {CapG-eGFPnuc }} \approx$ $21.2 \pm 0.2 \mu \mathrm{m}^{2} / \mathrm{s}$. Given the uncertainty of the estimate and the intrinsic accuracy of the FCS procedure, this is in very good agreement with the measured value.

The second component is characterized by its relative amplitude $\rho_{2}$ and diffusion time $\tau_{2}$. Data points with very large $\tau_{2}(>150 \mathrm{~ms})$, which seemed to be the result of cellular movement rather than protein diffusion, were excluded. Furthermore, exclusion of $\tau_{2}>150 \mathrm{~ms}$ from further analysis appears to be indicated because of statistical accuracy: as FCS is based on the statistical analysis of single fluctuations, the precision of FCS data is proportional to the amount of collected fluctuations. The longer the correlation times of underlying kinetic processes, the fewer corresponding fluctuations will be collected in

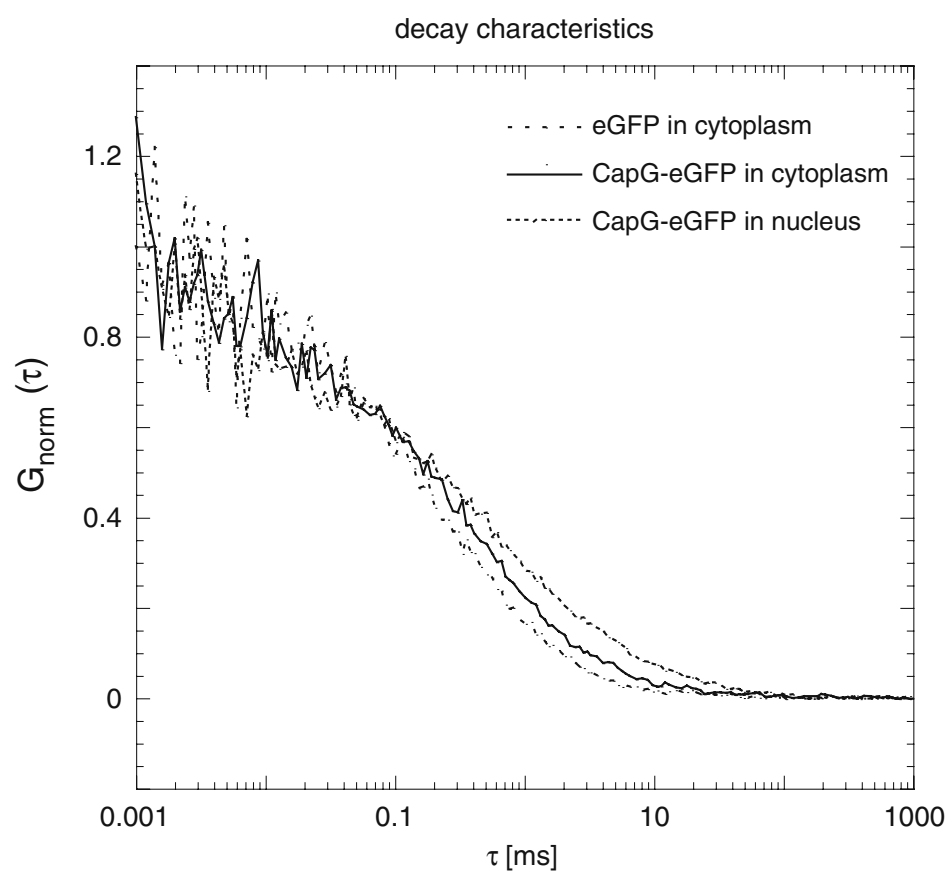

Figure 5. Normalized autocorrelation curves of cytoplasmic eGFP and nuclear and cytoplasmic CapG-eGFP: different decay characteristics are evident. 
the total run time $\left(T_{\mathrm{R}}\right)$ and the less precise will be the determination of the correlation time $\left(\tau_{\mathrm{d}}\right)$. According to a rough estimate, the relative error in the measurement is on the order of $N^{-1 / 2}$, where $N=$ $T_{\mathrm{R}} / \tau_{\mathrm{d}}$ (Qian et al. 1992); for example, in the case of a correlation time of about $800 \mathrm{~ms}$ and $60 \mathrm{~s}$ total run time, the relative error is about $12 \%$, whereas in case of $\tau_{\mathrm{d}}=150 \mathrm{~ms}$ in the same total run time it is only $5 \%$.

Amplitude $\left(\rho_{2}\right)$ and diffusion coefficients $\left(D_{2}\right)$ of the second diffusing component of CapG-eGFP in
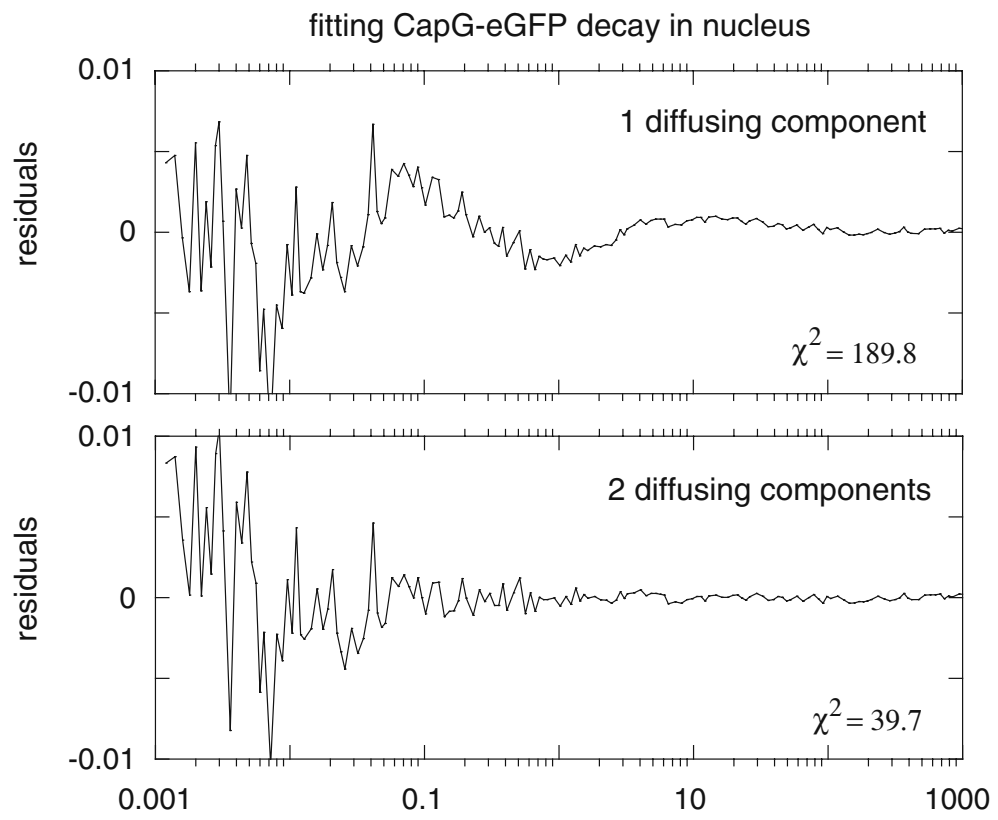

fitting CapG-eGFP decay in cytoplasm
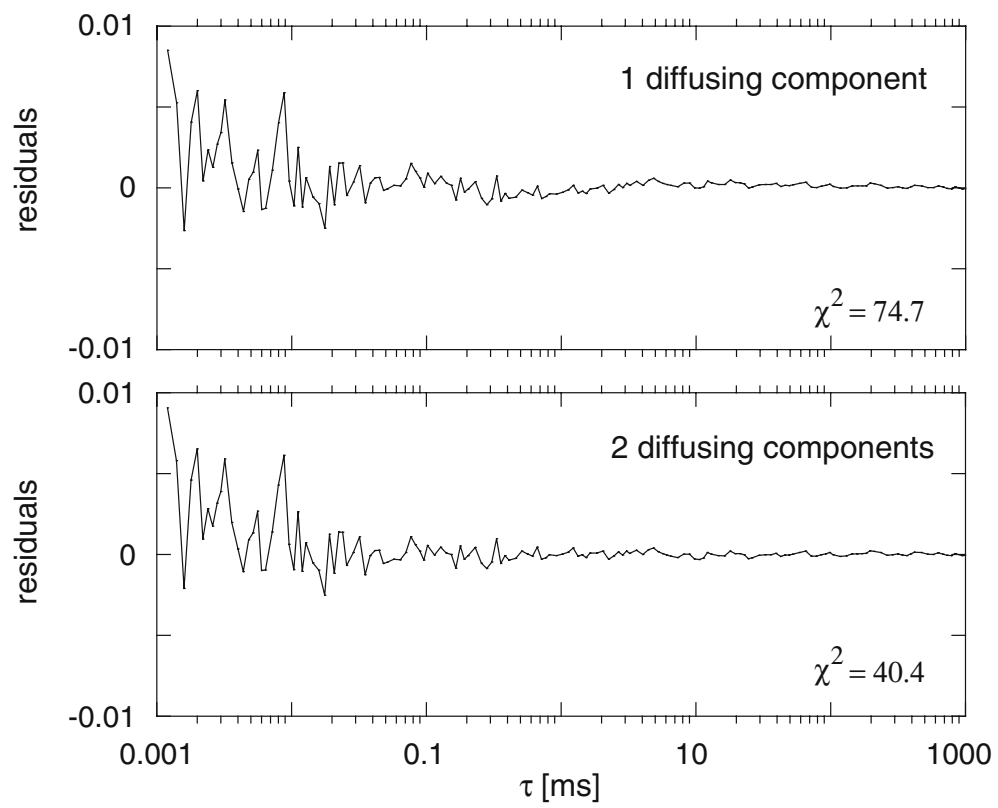

Figure 6. Residuals and $\chi^{2}$-values on fitting with one and two diffusing species in the nucleus and the cytoplasm, respectively: fitting two diffusing species distinctly improves fit results of nuclear and cytoplasmic CapG-eGFP. 
Table 2. Amplitude $\left(\rho_{2}\right)$ and diffusion coefficients $\left(D_{2}\right)$ of the second diffusing component CapG-eGFP in nucleus and cytoplasm of breast cancer cells

\begin{tabular}{lll}
\hline Parameter & Nucleus & Cytoplasm \\
\hline$\rho_{2}$ & $11.6 \pm 2.4 \%$ & $5.6 \pm 0.8 \%$ \\
$D_{2}$ & $0.27 \pm 0.04 \mu \mathrm{m}^{2} / \mathrm{s}$ & $0.20 \pm 0.02 \mu \mathrm{m}^{2} / \mathrm{s}$ \\
\hline
\end{tabular}

nucleus and cytoplasm of breast cancer cells are shown in Table 2.

Data analysis with $\tau_{1}$ fixed to the corresponding average value makes the differences in amplitude and diffusion coefficients of CapG-eGFP in nucleus and cytoplasm even more distinct (Table 3)

In both cellular compartments there exists a second slowly diffusing component of CapG-eGFP, but with different properties as revealed by FCS. In the cell nucleus, a larger fraction of CapG-eGFP is transiently immobilized to cellular structures or other partners of interaction. This is consistent with the decay characteristics of the normalized autocorrelation functions in Figure 5 and the worse fit results compared to the cytoplasm if only one diffusing component is considered (Figure 6).

The comparison of the average molecular brightness of the inert eGFP and the CapG-eGFP fusion protein makes a rough determination of the association status possible:

$$
\begin{aligned}
<\mathrm{F}_{\mathrm{eGFP}}>/<\mathrm{N}_{\mathrm{eGFP}}>=(2.0 \pm 0.04) \times 10^{3} \mathrm{cps} / \text { molecule } \\
<\mathrm{F}_{\text {CapG-eGFP }}>/<\mathrm{N}_{\text {CapG-eGFP }}>=(1.8 \pm 0.04) \times 10^{3} \mathrm{cps} / \\
\text { molecule in the } \text { nucleus } \\
<\mathrm{F}_{\text {CapG-eGFP }}>/<\mathrm{N}_{\text {CapG-eGFP }}>=(1.9 \pm 0.04) \times 10^{3} \mathrm{cps} / \\
\text { molecule in the cytoplasm }
\end{aligned}
$$

In general, the CapG-eGFP appears to diffuse as a monomer in the nucleus and the cytoplasm, which is in good agreement with reported results of gel filtration (Southwick \& DiNubile 1986), immunoprecipitation (Prendergast \& Ziff 1991) and ultracentrifugation (Zhang et al. 2006).

Table 3. Amplitude $\left(\rho_{2}\right)$ and diffusion coefficients $\left(D_{2}\right)$ of the second diffusing component CapG-eGFP in nucleus and cytoplasm of breast cancer cells with $\tau_{1}$ fixed to the corresponding average value

\begin{tabular}{lll}
\hline Parameter & Nucleus & Cytoplasm \\
\hline$\rho_{2}$ & $12.0 \pm 1.3 \%$ & $5.3 \pm 0.6 \%$ \\
$D_{2}$ & $0.38 \pm 0.06 \mu \mathrm{m}^{2} / \mathrm{s}$ & $0.25 \pm 0.04 \mu \mathrm{m}^{2} / \mathrm{s}$ \\
\hline
\end{tabular}

\section{Discussion}

Spectroscopic methods of kinetic analysis-FRAP and FCS - were used to characterize the dynamic distribution of the CapG-eGFP fusion protein within cell nuclei of living breast cancer cells on different timescales, i.e. in the microsecond to minute range. The CapG protein seems to be monomeric, and a large fraction diffuses freely in the nucleus. The diffusive behaviour of this fraction is mainly determined by its molecular size and the temperature and viscosity of the surrounding nucleoplasm and corresponds roughly to what was expected compared with the molecular size and measured diffusion time of eGFP alone. A smaller fraction of about $12 \%$ appears to be slowed down in diffusion to a hundredth of its regular mobility. The reason of this decreased mobility is hypothetical: the protein could be transiently attached to mobile or immobile interaction partners - such as polymerized nuclear actin. The different mobility in nucleus and cytoplasm hints at different kinds of transient CapG binding in these cellular sub-compartments.

Little or nearly none $(0-3 \%)$ of the CapG protein in the cell nucleus is immobilized on the timescale of minutes or even permanently. It shuttles rather rapidly within the nuclear compartment of breast cancer cells. The nuclear envelope, however, marks a distinct diffusion barrier: the nucleo-cytoplasmic shuttling takes a few minutes in breast cancer cells.

Applying FRAP and FCS as complementary spectroscopic techniques, it is possible to create a picture of a protein's dynamic distribution on different timescales. Although basically descriptive, these spectroscopic methods seem to be able to grant an alternative, broader view of a protein's properties. Studying protein dynamics inside the living cell provides an analytical tool to elucidate the spatiotemporal organization of cell nuclei, which may provide insights beyond mere description. In this context, the comparative FRAP analysis of CapG distribution in normal and neoplastic breast epithelial cells from our recent work (Renz et al. 2007) may serve as an example: The CapG protein appears to be transported much faster into the nucleus of breast cancer cells and is far less immobilized in the nucleus of cancer cells than in normal breast epithelial cells on the timescale of minutes. Both the immobilized fraction of CapG within the nucleus and its import kinetics distinguish normal and cancer 
cells independently. Comprehension of the dynamic distribution of CapG, especially its shuttling between different cellular compartments, might help to provide insight into its function in the context of motility, invasiveness and metastasis and might give clues to the altered cellular function in malignant dedifferentiation.

\section{References}

Axelrod D, Koppel DE, Schlessinger J, Elson EL, Webb WW (1976) Mobility measurement by analysis of fluorescence photobleaching recovery kinetics. Biophys $J$ 16: 1055-1069.

Dahl E, Sadr-Nabavi A, Klopocki E et al. (2005) Systematic identification and molecular characterization of genes differentially expressed in breast and ovarian cancer. $J$ Pathol 205: 21-28.

De Corte V, Van Impe K, Bruyneel E et al. (2004) Increased importin-beta-dependent nuclear import of the actin modulating protein CapG promotes cell invasion. J Cell Sci 117: 5283-5292.

Elson EL (1986) Fluorescence photobleaching and correlation spectroscopy for translational diffusion in biological systems. Biochem Soc Trans 14: 839-841.

Elson EL, Qian H (1989) Interpretation of fluorescence correlation spectroscopy and photobleaching recovery in terms of molecular interactions. Methods Cell Biol 30: 307-332.

Gettemans J, Van Impe K, Delanote V, Hubert T, Vandekerckhove $\mathrm{J}$, De Corte V (2005) Nuclear actin-binding proteins as modulators of gene transcription. Traffic 6: 847-857.

Goerisch SM, Wachsmuth M, Toth KF, Lichter P, Rippe K (2005) Histone acetylation increases chromatin accessibility. J Cell Sci 118: $5825-5834$.

Lippincott-Schwartz J, Snapp E, Kennworthy A (2001) Studying protein dynamics in living cells. Nat Rev Mol Cell Biol 2(6): 444-456.

Lu PJ, Hsu AL, Wang DS, Yan HY, Yin HL, Chen CS (1998) Phosphoinositide 3-kinase in rat liver nuclei. Biochemistry 37: 5738-5745.

Onoda K, Yu FX, Yin HL (1993) gCap39 is a nuclear and cytoplasmatic protein. Cell Motil Cytoskel 26(3): 227-238.

Parikh SS, Litherland SA, Clare-Salzler MJ, Li W, Gulig PA, Southwick FS (2003) CapG(-/-) mice have specific host defense defects that render them more susceptible than CapG $(+/+)$ mice to Listeria monocytogenes infection but not to Salmonella enterica serovar Typhimurium infection. Infect Immun 71(11): 6582-6590.

Pellieux C, Desgeorges A, Pigeon CH et al. (2003) CapG, a gelsolin family protein modulating protective effects of unidirectional shear stress. J Biol Chem 278: 29136-29144.

Petersen NO, Elson EL (1986) Measurements of diffusion and chemical kinetics by fluorescence photobleaching recovery and fluorescence correlation spectroscopy. Methods Enzymol 130: 454-484.

Prendergast GC, Ziff EB (1991) Mbh1: a novel gelsolin/severinrelated protein which binds actin in vitro and exhibits nuclear localization. EMBO J 10(4): 757-766.

Qian H, Elson EL, Frieden C (1992) Studies on the structure of actin gels using time correlation spectroscopy of fluorescent beads. Biophys $J$ 63: 1000-1010.

Rabut G, Ellenberg J (2005) Photobleaching techniques to study mobility and molecular dynamics of proteins in live cells: FRAP, iFRAP, and FLIP. In: Goldman R, Spector DL, eds. Live Cell Imaging. A Laboratory Manual. New York: Cold Spring Harbor Press, pp. 101-126.

Renz M, Betz B, Niederacher D, Bender HG, Langowski J (2007) Invasive breast cancer cells exhibit increased mbility of actinbinding protein CapG. Int J Cancer 122(7): 1476-1482.

Silacci P, Mazzolai L, Gauci C, Stergiopulos N, Yin HL, Hayoz D (2004) Gelsolin superfamily proteins: key regulators of cellular functions. Cell Mol Life Sci 61: 2614-2623.

Southwick S, DiNubile MJ (1986) Rabbit alveolar macrophages contain a $\mathrm{Ca}^{2+}$-sensitive, 41,000-Dalton protein which reversibly blocks the "barbed" ends of actin filaments but does not sever them. J Biol Chem 261(30): 14191-14195.

Sun HQ, Kwiatkowska K, Wooten DC, Yin HL (1995) Effects of CapG overexpression on agonist-induced motility and second messenger generation. J Cell Biol 129(1): 147-156.

Thompson CC, Ashcroft FJ, Patel S et al. (2007) Pancreatic cancer cells overexpress gelsolin family capping proteins which contribute to their cell motility. Gut 56(1): 95-106.

Van Impe K, De Corte V, Eichinger L et al. (2003) The nucleocytoplasmic actin-binding protein CapG lacks a nuclear export sequence present in structurally related proteins. $J$ Biol Chem 278(20): 17945-17952.

Vukojevic V, Pramanik A, Yakovleva T, Rigler R, Terenius L, Bakalkin G (2005) Study of molecular events in cells by fluorescence correlation spectroscopy. Cell Mol Life Sci 62(5): 535-550.

Wachsmuth M, Waldeck W, Langowski J (2000) Anomalous diffusion of fluorescent probes inside living cell nuclei investigated by spatiall-resolved fluorescence correlation spectroscopy. J Mol Biol 298(4): 677-689.

Wachsmuth M, Weidemann T, Müller G et al. (2003) Analyzing intracellular binding and diffusion with continuous fluorescence photobleaching. Biophys $J$ 84(5): 3353-3363.

Weidemann T, Wachsmuth M, Knoch TA, Müller G, Waldeck W, Langowski J (2003) Counting nucleosomes in living cells with a combination of fluorescence correlation spectroscopy and confocal imaging. J Mol Biol 334(2): 229-240.

Witke W, Li W, Kwiatkwoski DJ, Southwick FS (2001) Comparisons of CapG and gelsolin-null macrophages: demonstration of a unique role for CapG in receptor-mediated ruffling, phagocytosis, and vesicle rocketing. J Cell Biol 154(4): 775-784.

Zhang Y, Vorobiev SM, Gibson BG et al. (2006) A CapG gain-offunction mutant reveals critical structural and functional determinants for actin filament severing. EMBO $J \mathbf{2 5}$ : 4458-4467. 